\title{
Evaluation and Design of a Small Portable EMG Amplifier with Potential RMS Output
}

\author{
Yoshihiro Shimomura ${ }^{11}$, Koichi Iwanaga2), Hajime Harada2) and Tetsuo Katsuura ${ }^{2)}$ \\ 1) Graduate School of Science and Technology, Chi ba University \\ 2) Faculty of Engineering, Chiba University
}

\begin{abstract}
A bstract. The present study attempted to design and evaluate a small portable electromyogram (EMG) amplifier that can output enhanced EMG and its root mean square (RMS) value. The production and design were of a laboratory scale without any special or high cost circuit construction. The designed amplifier was actually innovated according to the actual working conditions based on physiological anthropology. The present amplifier was compared with commercially available products and proved to be of practical use. The device was installed with a sufficiently small body depicting 8-channel variable gain AC amplifier and variable timewindow RMS-to-DC converter. The prototype was battery-driven and well-shielded to minimize external noise interference.
\end{abstract}

(Appl Human Sci, 18 (2): 61-67, 1999)

Keywords: surface EMG, RMS converter, amplifier

\section{Introduction}

To quantitatively determine the working condition or assess the musculoskeletal model of humans, the use of electromyogram (EMG) is a common practice (Delp et al., 1996; Harada et al., 1995; Bohlemann et al., 1994; Karlsson and Peterson, 1992; Moritani, 1993; Winter, 1990). However, EMG is composed generally of highfrequency random signals. As such, direct waveform per se analyses have not been attempted outside the wards. The EMG amplitude output from a muscle is proportional to the work-load exerted on the muscle. Therefore, treatments of the amplitude in fieldwork, etc in physiological anthropology and ergonomics have been important. In evaluation tests of handtools, etc, integrated EMG ( $\mathrm{EEMG)}$ ) after recording the original waveforms has extensively been employed (Harada et al., 1995, Bohlemann et al., 1994). The principle underlying iEMG is basically simple and is advantageous as it can easily be obtained in the software aspect. Furthermore, there are cases where the mean value of EMG amplitudes has also been exploited (Ohashi, 1995). In cases where responses of real time mean values via an electric circuit are required, care should be exercised with precision on all waveforms not guaranteed, as errors are enlarged when the crest factor is large or the duty ratio of pulses is small. The approach to acquire the envelope by generally using a DC method after attenuation of high-frequency component with a low-pass filter (LPF) includes either the use of a digital filter after recording or possible real time output via LPF of an electric circuit (A kazawa et al., 1988). In cases where EMG is used as a trigger signal to control ratch mechanisms (Neptune and Hull, 1992), synchronization through the combined use of LPF (cutoff frequency, $3 \mathrm{~Hz}$ ) with an ideal absolute value circuit to establish the envelope representing the activity level of the muscle is essential. In expressing EMG in the form of an envelope, it is sufficient to establish the state of muscular activities, although information on the frequency is bound to be lost. According to the sampling theorem, the maximum frequency of a signal has to be sampled more than 2 fold to reproduce the complete signal. Based on spectral analyses, the maximum frequency of surface EMG is generally in the order of $1 \mathrm{kHz}$. Therefore, it is essential that a sampling rate of more than $2 \mathrm{kHz}$ is employed in sufficient recording of EMG. However, problems often arise in constructing the measurement system at this rate in multichannel recording over a long period of time. As such, the reduction of information load on envelope expression can delete these problems.

As an approach to expressing EMG in the form of an envelope, the root mean square (RMS) conversion may be resorted to. RMS is defined by the following expression:

$$
\operatorname{RMS}(f(t))=\left(\frac{1}{T}{ }_{0}^{T} f(t)^{2} d t\right)^{\frac{1}{2}}
$$

where $f(t)$ and $T$ represent the input waveform and averaging duration, respectively. RMS expresses the effective value of $A C$ signals, and may be proper for expressing the amplitude of electric signals rather than integration or filter, etc. In addition, it has been demonstrated that the RMS approach is preferred to 
integration in EMG treatment (Basmajian and De Luca, 1985). The RMS value of EMG signals has previously been applied in evaluation methods involving basic research of the neuromuscular system, work-load on muscles and regulatory signals of artificial hand dynamics (Moritani, 1993; Johnson, 1982). Moreover, it is also advantageous that the effective value of aliasing noise in $A / D$ conversion can be quantified.

In recent years, it is possible to convert EMG data into RMS values based on previously recorded data with the use of software innovated as a result of technical development in personal computers. However, when the above-mentioned issues of the measurement system, time spent on computing the moving averages and monitoring waveforms during recording are taken into consideration, derivation of not only raw EMG signals but its RMS values coupled with the use of additional hardware are warranted. Current EMG amplifiers used in experiments relating to physiological anthropology and ergonomics are usually heavy with a large body, rendering them impractical for appropriation to actual working condition of humans. The present innovation focused on practical application, and attempted to globally evaluate and design a compact multichannel amplifier with concurrent output of RMS values derived from amplified EMG signals.

\section{Design Description}

\section{General design}

As use of our present innovation in physiological anthropology may include outdoor applications at certain sites where an AC source is not available, a reasonable power source such as that afforded by 4 units of LR6 type alkali-battery was designed. As a positive-negative voltage source is generally required to drive an amplification circuit, a DC-DC converter with - 5V output was installed in the circuit board. In addition, the function generator for calibration use was not installed in the circuit board to afford a compact portable device. The number of channels were usually designated as eight. To cutoff any possible noise interference, shield-wire specifically designed for such purposes was employed in making the EMG-input cable.

\section{EMG amplifier}

Although alternatives exist for procuring the output of EMG signals, the usual bipolar connection involving the inputs of differential amplifier was employed in this study. Various modes were implemented to enhance the sensitivity of the signal discriminator, etc in the differential amplification-type amplifier. According to Koh and Grabiner (1993), the double differential technique is more effective than the single differential technique in minimizing crosstalks in differential amplifiers for EMG application. In addition, Herzog et al. (1993) devised a unique telemetry system to incorporate a 2-step type differential amplifier that could procure a large common mode rejection ratio (CMRR). As the present study required a high signal discriminatory function, the double differential type was preferred. Furthermore, an instrumental amplifier (to be elaborated below) was employed in counteracting the large DC gradient generated at the electrodes against the earth level.

The input stage of whole EMG amplification circuit (Fig. 1) was designed with TLC27L4 which is the highly precise quad OP-AMP IC. Special care was given to the amplification circuit by using circuit components of the highest quality. The capacitors and resistors used were thus the plastic film type with little phase displacement and metal film precision type with limited noise, respectively.

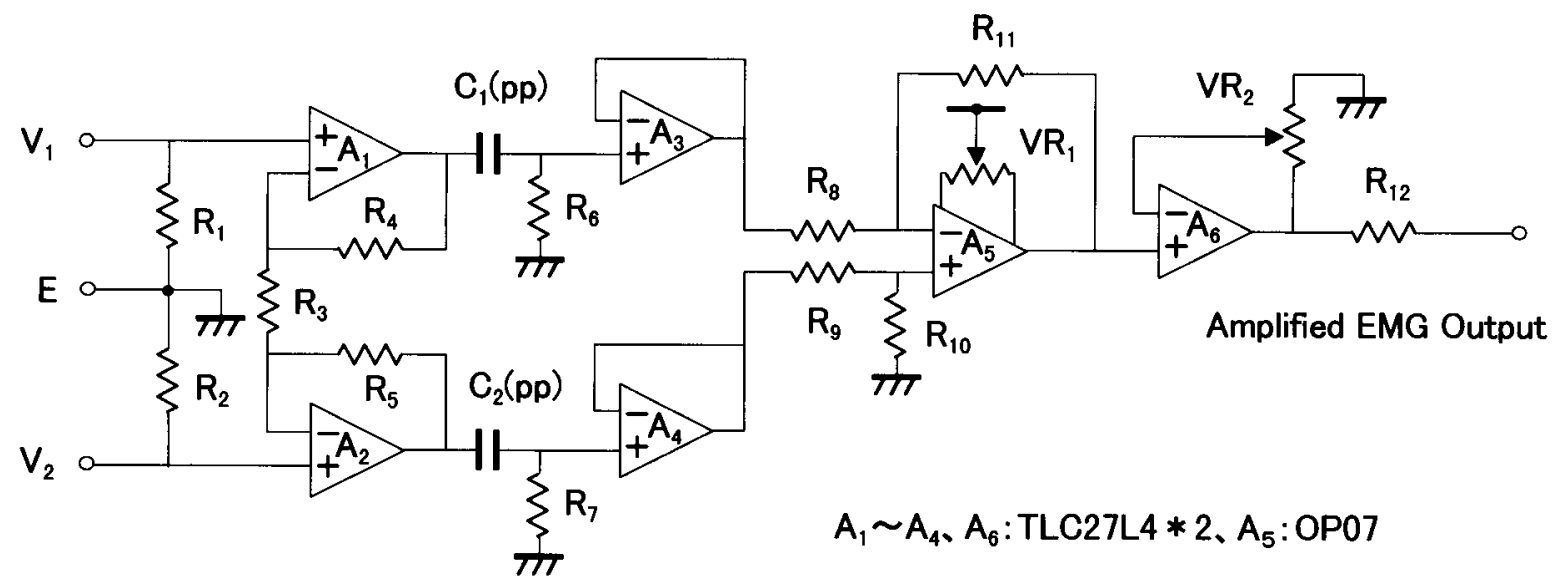

Fig. 1 The EMG amplification circuit. $V_{1}$ and $V_{2}$ are connected by two bipolar electrodes. $E$ is the earth terminal. The input impedance-deciding R1 and R2 measured $10 \mathrm{Mw}$. 
Amplification unit of $A_{1}$ and $A_{2}$ conduced Pseudodifferential amplification without changing the mean potential of $V_{1}$ and $V_{2}$ (Fig. 1). AC couplings induced by $A_{1}-A_{3}$ and $A_{2}-A_{4}$ nullified the $D C$ gradient generated by the state of electrodes. This AC coupling determined the time constant $(0.03 \mathrm{sec})$ of the performing EMG amplifier. The signal passing through the $A_{3}$ and $A_{4}$ buffer was input into the next-stage differential amplifier, $A_{5}$. OP07 IC was used in $A_{5}$ to perform offset adjustment. As output error would be exacerbated by having a large gain in the element corresponding to $A_{5}$ in the instrumental amplifier, the gain should be within a range of several multiples. A large gain was established at the input stage and a stage next to $A_{5}$. $A_{6}$ was a variable gain buffer, and adjustments of the output signal were achieved through $V_{2}$. The output of $A_{6}$ was derived after passing through the output protective resistor, $R_{12}$.

\section{RMS-to-DC converter}

Although several models of the RMS-to-DC converter were available on the market, a candidate with high precision low-cost with minimal components, AD736 (Analog Devices, 1997-1998), was selected. A smoothing capacitor was selectively employed for RMS calculation IC (Yates and Hertel, 1990). With regard to the RMS-toDC converter stage (Fig. 2), the averaging time of true RMS values was determined by only the smoothing capacitor $C_{A V}$. A $C_{A V}$ with a large capacity was vital in maintaining the rectified signal as it would elicit a direct effect on the calculation accuracy. The $C_{A V}$ therefore was accorded with a tantalum capacitor which had highperformance of impedance and thermal characteristics. Since the internal resistor of $I C$ combined with $C_{F}$ to function as an LPF, rippling effects of output were suppressed. Although the default value of $C_{A V}$ in the present amplifier registered $47 \mathrm{mF}$, alterations were

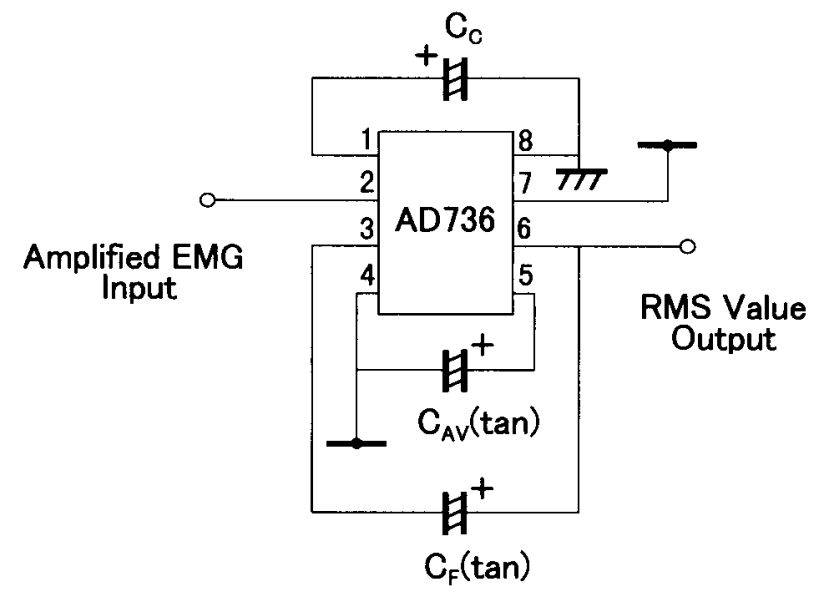

Fig. 2 True RMS-to-DC conversion circuit. The power source of $-5 \mathrm{~V}$ is shared by OP-AMP ICs. obtained with replacements. The input coupling capacitor, $\mathrm{C}_{\mathrm{C}}$, determined the lower range of frequency reduction of - $3 \mathrm{~dB}$. Since a time constant of 0.03 was designated in the EMG amplification circuit, $\mathrm{C}_{\mathrm{C}}$ was established at $10 \mathrm{mF}$ to acquire less than half value of the high-pass filter.

\section{Housing design}

The complete dimensions of the present innovation measured $40 \mathrm{~mm} \cdot 150 \mathrm{~mm} \cdot 200 \mathrm{~mm}$ (excluding protrusions) and weighed a mere $790 \mathrm{~g}$. Batteries, variable resistance knobs and power source indicator LED (green-yellow-red) were installed on top of the aluminium case accommodating the complete circuit. A screw terminal for input connector with two D-sub9pins (female) for output connector indicating amplified EMG and the RMS values were affixed.

\section{Evaluation}

\section{General procedure}

The evaluation procedure for the present innovation was generally reproducible in any standard laboratory designed for studies on physiological anthropology. An identical signal was separately input into the present innovation and another commercially available device, and the outputs were compared and contrasted. The MP100 system (Biopac System Inc.) was employed in data recording and analysis. The system was characterized with an input range of $-10 \mathrm{~V}, 16 \mathrm{bit}$ resolution and $2 \mathrm{kHz}$ sampling rate. Although the MP-100 system was originally designed as a routine network for measuring biosignals, it could also be employed in the present study by direct linkage with the analog input terminal. The three items used for analysis encompassed the output of $A_{6}(S D)$ in the circuit plan (Fig. 1), telemetry products such as NEC MT11 (TM) and the replaceable rack \& module type products such as Nihon Kohden AB-621G (NK). Cable, connector, installation equipment, etc were simulated according to the typical surface EMG experimental conditions. Standardized cables for surface EMG use were installed in all signal input lines, and all lines were connected to standard earth terminals while maintaining a clean surrounding electric environment. Differential input terminals (terminals used for surface electrodes) were shortened to minimize the output, or to minimize noise interference into the signal route, by securing appropriate routing of device placements and signal lines. On switching the power source on, a lapse of 30 min was allowed for the circuit to acquire a thermally stable condition before any measurements were initiated.

The amplifier set-up designated a time constant of 0.03 with the high cut filter off (i.e., upper limit of hardware) and notch filter for the noise of AC power source off. During experimental preparations and data recording, the output of amplifiers were monitored via 
Table 1 Experimental results

\begin{tabular}{ccccc}
\hline Amplifier & Common model $[\mathrm{mV}]$ & Noise $[\mathrm{mV}]$ & Phase shift $[\mathrm{msec}]$ & Time constant \\
\hline TM & $7.81-0.22$ & $7.68-0.07$ & $3.156-0.023$ & $0.6281-0.0065$ \\
NK & $4.46-0.16$ & $4.28-0.02$ & $0.005-0.015$ & $0.6150-0.0035$ \\
SD & $4.34-0.02$ & $3.23-0.02$ & $0.021-0.025$ & $0.6344-0.0025$ \\
\hline
\end{tabular}

Mean - SD. TM, NK, SD indicated amplifiers tested in this experiment. They were telemetry products, rack $\&$ module type products, and output of $A_{6}$ in our circuit plan respectively. Common mode and noise indicated mean effective value of amplifier output potential; phase shift represented the time delay against the standard wave; and time constant demonstrated the attenuation rate of amplifier output potential within $30 \mathrm{msec}$.

the oscilloscope, confirming high-frequency noise was not interfering with the output or that it was negligibly small even when encountered. For the amplifier that indicated a large DC offset, zero adjustment was corrected. After appropriating calibration, five experiments were conducted. Each experiment lasted $30 \mathrm{sec}$ with the frequencies of two standard waves measuring $43 \mathrm{~Hz}$ and $2.6 \mathrm{~Hz}$.

\section{Calibration}

A calibrator was assembled from the function generator IC, ICL8038. The calibrator was a CR timer and furnished 4-wave output with squared/triangular waves of respective inverse/non-inversed positions and variable amplitude, duty ratio and frequency. In addition, an approximately 1000:1 attenuator was installed in the calibrator. The output of squared waves (approx., $2 \mathrm{mVp}$ p) of the calibrator was subjected to differential inputs on inversed phase to output amplitudes. The amplitudes were monitored and then coordinated to an identical gain (sensitivity) among the three amplifiers, resulting in a gain of approximately 1000 fold. This gain was fixed throughout the experiments thereafter.

\section{Common mode test}

Triangular waves from the calibrator were input on non-inversed phase into the respective amplifiers at 43 $\mathrm{Hz}$, and the mean effective value of output potentials of amplifiers was derived. The results are indicated in Table 1. As factors such as the routing of signal lines, positions of electric devices and absence/presence of humans in the vicinity induced slight variations in input potential, the value obtained was an indication. This should, however, be a measure to ensure the function of amplifiers in realizing minimum valuation. NK and SD were of the same degree, although TM manifested a significantly high value. This trend was constantly observed even when monitoring the waveforms. With regard to the results of FFT (Fig. 3), the frequency ( 43 $\mathrm{Hz}$ ) of standard wave corresponded well to average condition of other parts, rendering nullification of common mode signals without any problem. CMRR of the present innovation definitely approximated $60 \mathrm{~dB}$, even in

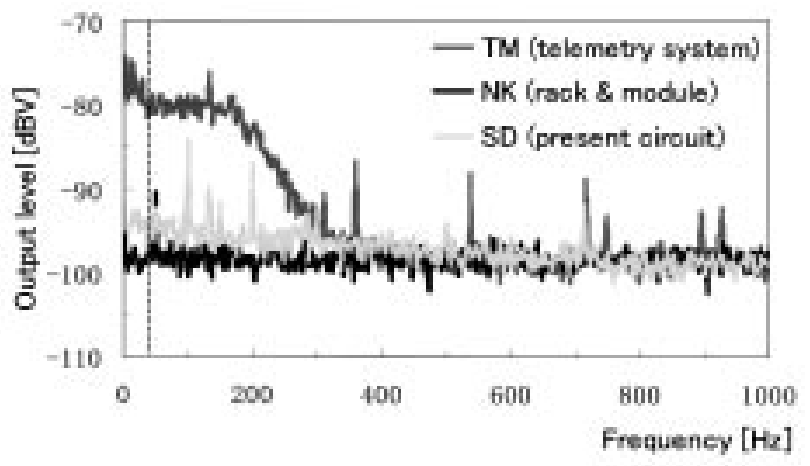

Fig. 3 Results of FFT in Common mode testing, where $F s=2000$ $\mathrm{Hz}, \mathrm{N}=4096, \mathrm{Navg}=8$ and window $=\mathrm{Hanning}$. Dotted line represents $43 \mathrm{~Hz}$ of standard wave. TM, NK, SD indicated amplifier types tested in this experiment.

an experimental environment where EMG determination with a constant noise interference was conducted (without earth connection). As clearly demonstrated in the observed waveform, low-frequency noise in TM was especially large. This was probably attributed to noise generated by imprecise contact points and electrical maladjustment within the system. Furthermore, TM clearly shifted a level on $200 \mathrm{~Hz}$ regardless of the high cut filter being off. This was due to the frequency specificity of $0.1-200 \mathrm{~Hz}$ of the product used.

\section{Noi se test}

By shortening the respective differential input connectors of amplifiers, the mean effective value of output potentials was derived (Table 1 ). The effective values significantly ranked in a descending order with TM NK SD. The major culprit was low-frequency noise. Results of FFT (Fig. 4) indicated that interference of noise derived from the commercial $50 \mathrm{~Hz}$ power source in all amplifiers registered values of the same order. As for the qualitative aspect, it was characteristic that the $50 \mathrm{~Hz}$ power did not show any increases even when it was not earthed because the system was electrically closed beside having battery-driven SD. With regard to NK, the 0 adjustment might have been deficient. 


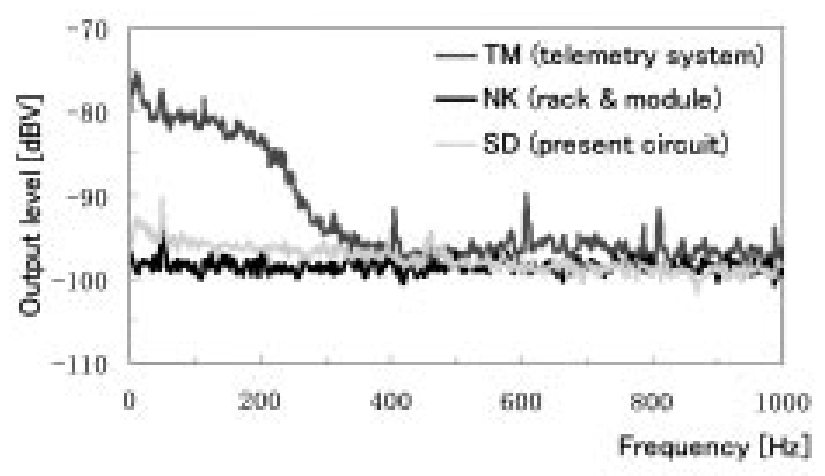

Fig. 4 Results of FFT in noise testing, where $F s=2000 \mathrm{~Hz}$, $\mathrm{N}=4096, \mathrm{Navg}=8$ and window $=\mathrm{H}$ anning. $\mathrm{TM}, \mathrm{NK}, \mathrm{SD}$ indicated amplifier types tested in this experiment.
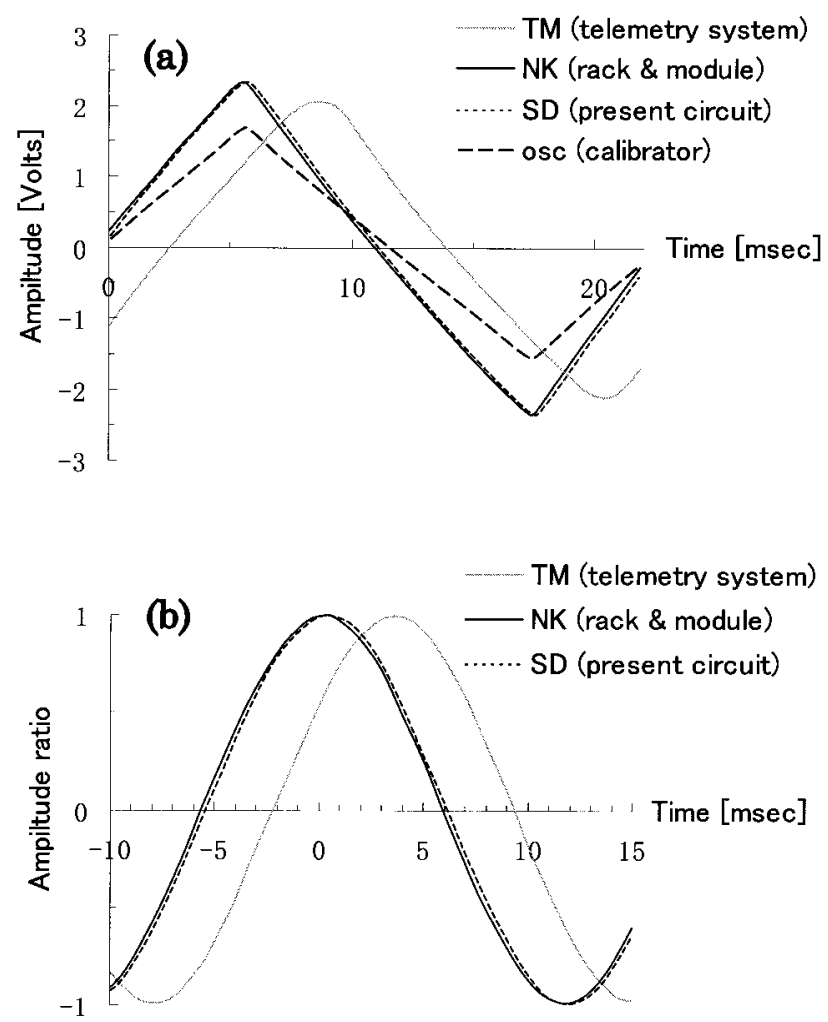

Fig. 5 (a) An example of an actually measured wave in a phase shift test conducted. Osc represents a standard wave of calibrating function generator before attenuation. (b) Cross correlation function against standard wave in the phase shift test.

\section{Phase shift test}

Triangular waves of calibrator were inversed phase input into the respective amplifiers at $43 \mathrm{~Hz}$ before comparing the peak displacement time against the standard wave. Results of the measured waveform (Fig. 5 (a)) are illustrated in Table 1. It is clear from the cross

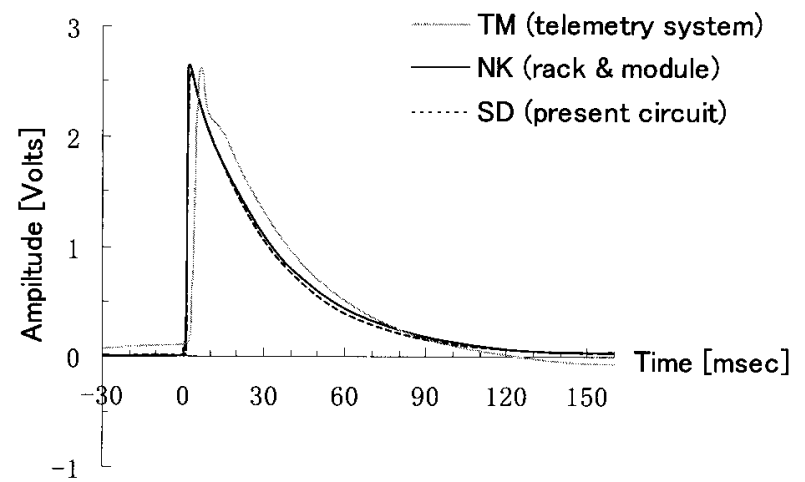

Fig. 6 An example of an actually measured waveform in a time constant test conducted.

correlation function of Fig. 5 (b) that little or no phase displacement was observed between NK and SD in practice. From the actual EMG tests shown later, the 3 msec displacement of TM persistently prevailed without any dependence on the frequency. As such, this was considered as time delay attributed to multiplex and transmission, etc of signals. As is clearly shown in Fig. 5 (a), the edge of waveform blunted by cutoff frequency due to the use of $200 \mathrm{~Hz}$ LPF was distinctly displayed.

\section{Time constant test}

Squared waves of calibrator were inversed phase input into the respective amplifiers at $2.6 \mathrm{~Hz}$ before comparing with the attenuation time of output. All the time constants of amplifiers were designated at $\mathrm{rc}=0.03$, a value that has often been used in EMG measurements. As such, the attenuation rate within $30 \mathrm{msec}$ has extensively been known from the following expression:

$$
\begin{aligned}
& \mathrm{T}=-\mathrm{rc} \ln \left(1-\mathrm{V}_{\text {out }} / \mathrm{V}_{\text {source }}\right) \\
& \mathrm{V}_{\text {out }} / \mathrm{V}_{\text {source }}=0.6321
\end{aligned}
$$

With the actually measured waveform (Fig. 6) and attenuation rate (Table 1 ) illustrated, both TM and SD indicated a comparable theoretical value. Through the attenuation process of post-edging, both NK and SD portrayed a smooth CR curve theoretically. However, open spaces signifying inappropriate overlapping of $C R$ plot during the early attenuation stage for TM were observed. Furthermore, attenuation persisted even when the GND level was surpassed, filter treatment other than global functions was warranted. The slow onset of TM was due to integration activities by the $200 \mathrm{~Hz}$ LPF.

\section{Actual EMG test}

The input cable of each electrode was branched to conduct a common EMG potential by connecting respectively to the amplifiers. Based on high-amplitude surface EMG procurable through experience, the $\mathrm{m}$. 

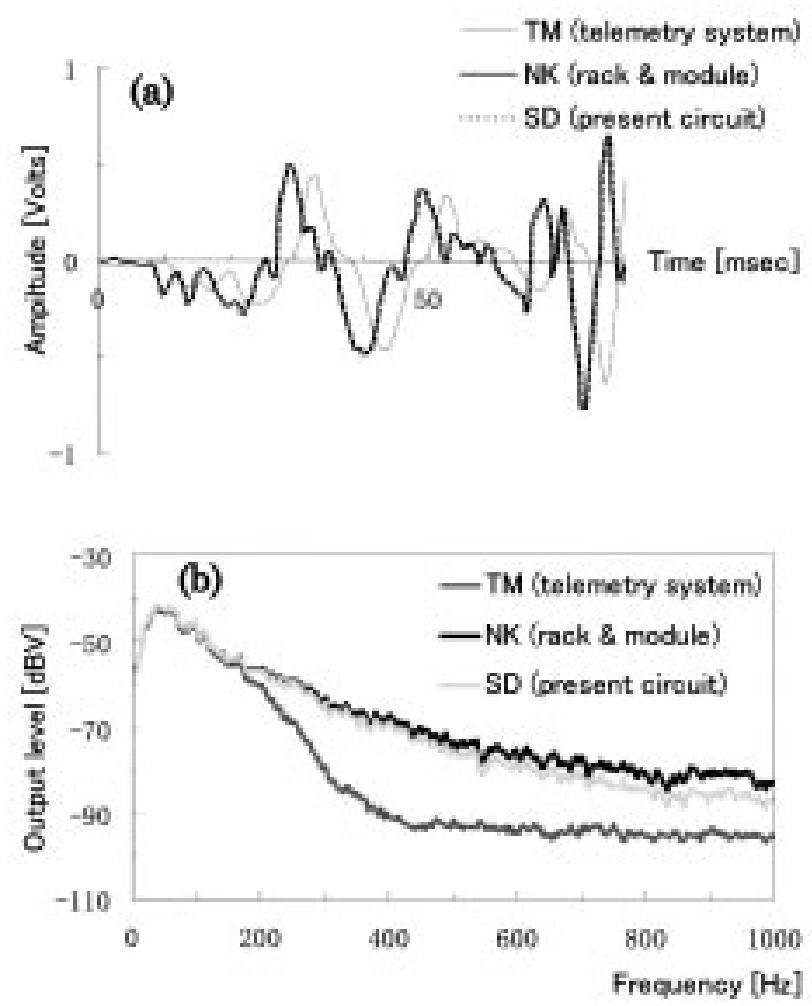

Fig. 7 (a) An example of an actually measured waveform of an actual EMG test conducted. (b) FFT results of the actual EMG test, where $\mathrm{Fs}=2000 \mathrm{~Hz}, \mathrm{~N}=4096, \mathrm{Navg}=8$ and window $=$ Hanning.

biceps brachii were selected as the sites for recording EMG potentials. Disposable Ag-AgCl surface electrodes with interelectrode impedance of about $13 \mathrm{kw}$ were used. As the input impedance of amplifiers far exceeded the interelectrode impedance, it was therefore ignored. Qualitative analysis of the actually measured waveforms was conducted by appropriating movement to muscle samples at $0-100 \%$ MVC. With the typical example of an actually measured waveform (Fig. 7 (a)) and results of FFT (Fig 7 (b)), real time outputs of the respective amplifiers could not be discriminated by the naked eye. However, when waveform displacements induced by LPF and $3 \mathrm{msec}$ delay were detected, identification would then be secured. Because the delays and differences in spectrum between NK and SD were negligible, discrimination was difficult even after post-recording analysis. When the time constant was 0.03 , the cutoff frequency approximated $5.3 \mathrm{~Hz}$ as a result of RC coupling in the electronic circuit. The results of FFT confirmed the low frequency attenuation in all amplifiers.

\section{Discussion}

The special features of the amplifier innovated in this study did not differ much from commercially available devices, especially product NK that was composed of an discrete circuit construction. Moreover, the innovation tolerated sufficient practical use. However, in the "practicality" aspect, the innovation was not merely limited to applicability under an ideal condition. In fact, when using ME device, we are often plagued by problems with regard to setting up the devices. For instance, in the case of systemic construction in experiments at sites outside the laboratories, certain actual issues involving material transport, electricity source, earth line and noise cut-down require a great deal of time and effort. Size reduction, battery-powered and complete shielding of the system of the present amplifier were factors that resolve these problems. Moreover, BNC connecting adapter and universal multipurpose connector were installed in cable types, facilitating application of the innovation. The present innovation has been employed in several experiments, and its efficacy was non-disputable.

Envelope output contributed to not only compactness but practical waveform monitoring as well. For instance, when EMG of $\mathrm{m}$. tibialis anterior is measured during maximum voluntary contraction (MVC), and then the direction of load weighing on the dorsalis pedis is qualitatively determined, it is doubtful if the test muscle did actually yield the MVC at all. At this juncture, EMG was quantitatively measured, and the direction of load was determined when envelope output was used, rendering EMG measurements possible at real MVC. By using the RMS value in an envelope in the case where EMG was considered as an electrical signal (regardless of $A C$ or DC), comparative studies of all voltage waveforms and theoretically the most reliable are possible (Analog Devices, 1997-1998). Furthermore, as simple amplification of EMG and its RMS value can concurrently be obtained, raw EMG recording was possible while monitoring the envelope.

With regard to calculation precision of RMS value of IC (Analog Devices, 1997-1998) used in this amplifier, for instance, the region of $1 \%$ error reading at $10 \mathrm{mVrms}$ of input amplitude easily surpassed the $2 \mathrm{kHz}$ upper limit of EMG (Winter, 1990). Furthermore, although the actual measured value of crest factor of EMG exceeded by 2-3, the waveform of crest factor 5 could even be suppressed to errors of within a few percent. When these are considered in actual use, the accuracy is sufficiently high.

The most problematic disadvantage of the present amplifier is the mere installation of secondary LPF as the antialiasing filter. Therefore, in cases where recording of the raw $E M G$, if the $A / D$ converter was to perform at least at a speed exceeding $1 \mathrm{kHz}$, the higher frequency band of EMG per se became noise. However, as the system can strongly tolerate noise, aliasing noise from the exterior was not observed. A LPF with sufficiently high function would be installed in future. 
As for costing, such as components consisting of the EMG amplification unit, RMS calculation unit, power source unit and other relevant parts amounted to $¥ 50,000$. This was $1 / 10$ the cost of commercial products, and it was comparatively economical when viewed on the functionality aspect. However, the present amplifier was not innovated as an industrial standard product. Viable cost-cutting may be attained by further adjustment of components other than electrical parts such as connector, casing, etc.

\section{Conclusion}

The present study designed a small, portable amplifier that afforded amplified EMG with concurrent output of its RMS value. The designed amplifier, manufactured at low cost, indicated a accuracy comparable to those of commercial products. This amplifier is small and noise-tolerant as it is batterydriven. As such, the present innovation can be employed in fieldwork related to physiological anthropology.

Acknowledgement. The authors are grateful to the laboratory staff for their excellent technical expertise and efficient manufacturing assistance.

\section{References}

Akazawa K, Takizawa H, Hayashi Y, Fujii K (1988) Development of control system and myoelectric signal processor for bio-mimetic prosthetic hand. Biomechanism 9: 43-53. Tokyo univ press.

Analog Devices (1997-1998) Linear data book

Basmajian JV, De Luca CJ , ( 1985) Muscles alive: Their function revealed by electromyography. 5 th ed. Williams and Wilkins, Baltimore

Bohlemann J, Kluth K, Kotzbauer K, Strasser H (1994) E rgonomic assessment of handle design by means of electromyography and subjective rating. Appl Ergonomics 25 (6): 346-354

Delp SL, Grierson AE, Buchanan TS (1996) Maximum isometric moments generated by the wrist muscules in flexion-extension and radial-ulnar deviation. J Biomechanics 29 (10) : 1371-1375
Design Wave (1997) The linear IC manual (Analog function IC). CQ press, Tokyo

Harada H, Katsuura T, Kikuchi Y ( 1995) Comparative study on the muscular load of the arms using hair driers. J Human Ergol 24: 183-192

Herzog W, Stano A, Leonard TR (1993) Telemetry system to record force and EMG from cat ankle extensor and tibialis anterior muscles. J Biomechanics 26 (12) : 1463 1471

J ohnson B (1982) Measurement and evaluation of local muscular strain in the shoulder during constrained work. J Human Ergol 11: 73-88

Karlsson D, Peterson B (1992) Towards a model for force predictions in the human shoulder.J Biomechanics 25 (2): 189-199

Koh TJ , Grabiner MD ( 1993) Evaluation of methods to minimize cross talk in surface electromyography. J Biomechanics 26 (suppl 1) : 151-157

Moritani T (1993) Neuromuscular adaptations during the acquisition of muscle strength power and motor tasks. J Biomechanics 26 (suppl 1): 95-107

Neptune R, Hull ML (1992) A new electromechanical ski binding with release sensitivity to torsion and bending moments transmitted by the leg. Int J Sport Biomechanics 8: 331-349

Ohashi J (1995) Differences in changes of surface E MG during low-level static contraction between monopolar and bipolar lead. Appl Human Sci 14 (2): 79-88

Suzuki M (1996) The monolithic OP AMP manual. CQ press, Tokyo

Winter DA(1990) Biomechanics and motor control of human movement. 2nd ed. John \& Sons, New York

Yates JW, Hertel SA (1990) Computer-based analysis of EMG signals: design and use of an RMS-to-DC converter. Computer Aided Ergonomics, Tylor \& Francis, 490-498

Received: J anuary 5, 1999

Accepted: February 16, 1999

Correspondence to: Yoshihiro Shimomura, Department of E rgonomics, Faculty of Engineering, Chiba University, 1-33, Yayoi-cho, Inage-ku, Chiba-shi, Chiba 263-8522, Japan 